\title{
Prototipo de sistema de recomendación grupal en un destino turístico
}

\author{
Pedro Vargas Pérez \\ Hilton Birmingham Metrople \\ pedro.vargasperez@hilton.com
}

José Luis Leiva Olivencia
Universidad de Málaga
jlo@uma.es

Recibido: 11-06-2015

Aceptado: 02-07-2015

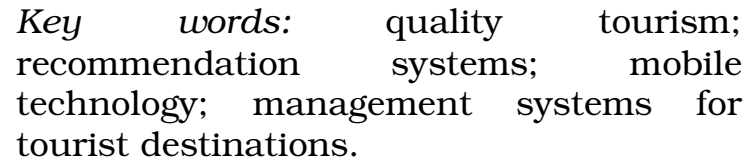

\section{ABSTRACT}

Prototype for group recommendation systems in a destination. ICT is a very active field to provide added value to visits in a destination for a group of tourists. The destinations should offer technological tools able to give personalized information, interactive, etc... Nowadays, the use of tools like guides or websites covers the tourists' basic needs, however, they are insufficient, because the evolution of mobile devices have induced a change in the way the tourists interact and obtain the information.

A special case is the use of recommendation systems in mobile devices, what allows the apps developing which enriches the tourist experience. In this scope, it has a special relevance the personalization of the information through the group recommendation systems. This article show a prototype which uses recommendation techniques for group visits in an integrated system of destination management.
Palabras clave: calidad turística; sistemas de recomendación; tecnología móvil; sistemas de gestión de destinos.

\section{RESUMEN}

Las tecnologías de la información y las comunicaciones constituyen un campo muy activo para ofrecer un valor añadido a las visitas a un destino de un grupo de turistas. Los destinos deben proporcionar herramientas tecnológicas capaces de ofrecer información personalizada, interactiva, etc. Actualmente es muy común el uso de herramientas como guías o páginas web que cubren las necesidades básicas de los turistas, pero, se tratan de recursos insuficientes, pues la evolución de los dispositivos móviles ha provocado un cambio en la forma en que los turistas interactúan y obtienen información.

Un caso concreto es la utilización de sistemas de recomendación en dispositivos móviles, que permite desarrollar aplicaciones que enriquecen la experiencia turística. En este ámbito, resulta de especial interés la personalización de la información a través de sistemas de recomendación grupales. Se presenta en este trabajo un prototipo que utiliza técnicas de recomendación para visitas en grupo en un sistema integrado de gestión de destinos (SIGD).

\section{INTRODUCCIÓN}


El turismo supone dentro de la economía de los países un papel muy importante (UNWTO, 2015), ya sea como:

- Fuente de ingresos producidos por los clientes $^{1}$ que visitan los puntos de interés turisticos.

- Elemento de fomento del sector turistico en los países de origen. Empresas del sector que dirigen, aconsejan o simplemente venden destinos turísticos. (agencias de viaje, compañias aéreas, cadenas hoteleras, ...).

- Motor de desarrollo de nuevas tecnologias que ayudarán en la selección de destinos así como de los POI's a visitar durante la estancia en un determinado destino turístico.

La importancia y el volumen económico que supone hasta ahora el turismo es sólo un indicativo de lo que puede llegar a suponer. Si nos fijamos en las previsiones que la organización mundial del turismo maneja, vemos que se trata de algo mucho más importante de lo que en un principio podríamos pensar.

El crecimiento continuo y casi exponencial que en las últimas décadas ha experimentado el turismo y la amplia diversificación en cuanto a destinos y sectores turísticos, nos hacen ver que se trata de un factor clave en el desarrollo socio-económico de carácter global (UNWTO, 2015).

El turismo supone un motor de desarrollo de nuevas tecnologías, y un ejemplo de aplicación turística de las tecnologías, es la utilización de sistemas de recomendación grupales (Leiva, 2014). Por otra parte, el desarrollo tecnológico y las nuevas posibilidades que se ofrecen hoy día, tanto en lo que a hardware y software se refieren, hacen que la selección de destinos turísticos y de los POI's a visitar dentro de un destino sean más fáciles y acertadas.

De este modo se consigue que el resultado de la visita a un destino, sea por lo general, más satisfactorio que en uno en el que no se cuente con estas herramientas.

No obstante, en los primeros estudios y pruebas realizados con estas herramientas tecnológicas aplicadas al turismo, encontramos como principal problema, el exceso de información aportada, siendo uno de los objetivos fundamentales de las herramientas actuales aportar POI's recomendados a un cliente (turista particular o grupo) los más próximos posibles a su preferencia y procurando que el número de resultados no sea excesivo ${ }^{2}$.

Entre los elementos que cabe destacar de entre los importantes desarrollos tecnológicos aplicados al turismo encontramos:

- Por una parte la aparición de dispositivos portátiles, tales como smartphones de última generación o tablets con una gran capacidad de procesamiento y con acceso a internet $^{3}$, supone uno de los pilares fundamentales en el uso de la realidad aumentada aplicada al turismo (Lymberopoulos et al., 2011).

- Por otro lado el software ya existente, capaz de almacenar el perfil del cliente y hacer una búsqueda de los POI's próximos al punto donde se encuentra el turista y que más se adaptan a sus preferencias. Por destacar alguno de los primeros en usar estas tecnologías, podemos hablar de: LAYAR o WIKITUDE (Leiva et al, 2012).

1 Normalmente nos referiremos al turista como cliente, usuario.

2 El exceso de información está muy cercano a la desinformación, debido a que el cliente no está dispuesto a perder excesivo tiempo analizando un gran número de resultados.

3 Ya sea mediante una red inalámbrica WiFi o con tarifa de datos proporcionada por algún operador de telefonía 
- La existencia de amplias BBDD con una extensa información de los POI's de determinados destinos turísticos, es también un elemento fundamental para que todas estas aplicaciones puedan funcionar.

Una de las aplicaciones móviles que están comenzando a utilizarse en destinos turísticos, son aquellas que están basadas en realidad aumentada (Leiva, 2014). La importancia de la realidad aumentada, no estriba sólo en la selección de los POI's, que supone un trabajo muy importante y un modo de facilitar la selección de destinos al cliente. El turista puede tener acceso a comentarios realizados por otros usuarios que han visitado con anterioridad esos POI's, además también puede permitirle experimentar simulaciones de eventos históricos, lugares y objetos (Lashkari et al, 2010).

Por supuesto la información que aporta un motor de recomendación a un sistema de realidad aumentada, no queda reducida al texto escrito o a la imagen visual, también aporta audio que en determinados contextos puede ser enriquecedor.

En la visita a un determinado POI, puede ser interesante, que contemos con un guía que nos acompañe o bien con una visión de cómo un determinado edificio era en su momento de más esplendor. También podríamos hacer una visita real al Teatro Romano, la Alcazaba y al Castillo de Málaga, pero con ayuda de la realidad aumentada seríamos capaces hacernos una idea de cómo eran en el pasado en base a las explicaciones que los expertos nos dan al respecto, pero además con las herramientas tecnológicas de las que disponemos hoy en día podríamos "verlo" tal y como fueron ${ }^{4}$ y en distintos momentos de la historia.

En el presente artículo se realizará un repaso de las diferentes técnicas de recomendación existentes, para profundizar en el diseño de un prototipo de sistema de recomendación grupal para un destino turístico. Para finalizar se abordarán unas conclusiones sobre la importancia que tendría la utilización de estos tipos de sistemas en un destino.

\section{SISTEMAS DE RECOMENDACIÓN}

Un sistema de recomendación, es aquel que teniendo en cuenta los intereses y preferencias de un usuario o grupo de estos, es capaz de seleccionar, de un conjunto de ítems, aquellos que se acercan más a los intereses del mismo. En este caso concreto trataremos al grupo como un único cliente, evidentemente una vez hayamos aplicado una serie de fórmulas y algoritmos para aconsejar al grupo como si de un individuo único se tratara, no obstante tendremos las preferencias ponderadas del conjunto de individuos (Leiva, 2014).

Básicamente, el turismo conlleva elección: del destino a visitar, del medio de transporte, de las actividades a realizar, del alojamiento que se va a usar...

Las tecnologías de la información incorporan una serie de ventajas frente a los medios de comercialización tradicionales que, entre otras, posibilitan la adecuación de cualquier servicio/producto a un cliente con especificaciones muy concretas.

Las herramientas que actualmente existen y que se aplican al turismo así como las que siguen surgiendo, ofrecen una gran diversidad de posibilidades al turista. Esta cantidad de información se vuelve en muchos casos excesiva, lo cual produce una sobrecarga de datos y por lo tanto que el usuario no se sienta atraído por el uso de la herramienta (Lymberopoulos et al., 2011).

Los usuarios estamos constantemente conectados buscando información de diverso tipo (correo, noticias, redes sociales, etc.). Pero Internet contiene tal sobrecarga de

4 Al menos ver una reconstrucción (o varias incluso) según las diferentes opiniones de los expertos 
información que, muchas veces, el usuario no sabe dónde ni cómo buscarla, ya que debe realizar un filtrado de la misma y localizar aquella que le sea de utilidad (Leiva et al, 2012).

A partir de esta necesidad surgen los sistemas de recomendación. Éstos realizan un filtrado de toda esa información disponible y nos dan aquellos resultados que consideran que pueden ser de interés para los usuarios, de forma que, nos facilitan la toma de decisiones sin gastar tiempo ni esfuerzo.

Dada la gran cantidad de oferta posible y para conseguir herramientas más útiles y atractivas al cliente, se hacen necesarios los sistemas de recomendación. Esta misma problemática se ha visto en otros sectores y no sólo en el turístico: música, comercio electrónico,... En cualquiera de estos sectores la cantidad de productos ofertados es tal que se hace necesario algún sistema de recomendación que según el perfil del usuario filtre o seleccione un conjunto suficientemente reducido de productos para que el cliente no se sienta desanimado o frustrado en su búsqueda.

El objetivo, por tanto, de un buen sistema de recomendación es hacer que el cliente no se sienta desbordado por la cantidad de información y que los resultados mostrados sean los más adecuados al perfil del individuo.

Hoy en día nos encontramos con sistemas de recomendación aplicados a muy distintos sectores, pero en todos ellos se siguen similares criterios procurando mostrar un conjunto suficientemente reducido de resultados al cliente que se base en las preferencias del mismo: iTunes, Spotify, Amazon, ...

\subsection{Ventajas e inconvenientes de los sistemas de recomendación}

Si bien en un principio podría parecer que el uso de sistemas de recomendación aporta sólo beneficios, encontramos también algunas desventajas (Leiva, 2014). A continuación enumeramos las ventajas e inconvenientes que pueden aportar la utilización en un destino turístico de un sistema de recomendación:

\subsubsection{Ventajas}

- El ofrecer al usuario un producto ya filtrado sin necesidad de realizar la búsqueda, permite el ahorro de tiempo y esfuerzo.

- Incrementa las ventas cruzadas al recomendar productos adicionales.

- Mejora la fidelidad de los usuarios, recomendando productos que el turista desea. Esta personalización exitosa provoca que estos sistemas posean un valor añadido.

\subsubsection{Inconvenientes}

- Los sistemas de recomendación están basados en algoritmos internos que pueden estar influenciados por intereses económicos y recomendar determinados productos en primera posición frente a otros que podrían ser de mayor interés para el turista.

- La dificultad de recomendar cuando tenemos un bajo número de usuarios que otorgan sus valoraciones o introducimos un nuevo producto en el sistema sin ninguna valoración.

- Falta de privacidad al permitir a la aplicación acceder a nuestros datos personales y preferencias introducidas.

- Los usuarios en principio no están motivados para cumplimentar sus preferencias en el sistema, lo que ocasiona que a veces haga falta usar incentivos. 
- El mantenimiento de un sistema de recomendación es bastante costoso. Por ello, es necesario recurrir a fuentes de financiación públicas (ayuntamientos, comunidades autónomas, diputaciones provinciales,...) o privadas (sponsor que publicite productos).

\subsection{Mecanismos de recomendación}

Aunque la variedad de sectores en los que se pueden aplicar mecanismos de recomendación es muy amplia, en este apartado nos vamos a referir sólo al ámbito turístico.

Para recomendar una actividad turística a un usuario, son aplicables varios tipos de técnicas: criterios relacionados con los POI's más visitados, tipos de visita que realiza habitualmente el visitante, información demográfica de los turistas,...

Si las recomendaciones obtenidas por un turista son buenas (desde el punto de vista del turista) potenciará la fidelización del uso del sistema de recomendación. En la medida en que un mismo turista vuelve a usar el sistema de recomendación éste puede tener más información del turista en cuestión y hacer recomendaciones más acertadas.

Veamos qué elementos debe contener un sistema de recomendación, a partir de los cuales podremos aplicar cualquiera de los distintos mecanismos de recomendación:

a) Datos de campo: conjunto de datos que el sistema debe poseer antes de que el proceso de recomendación comience.

b) Datos de entrada: conjunto de datos que el usuario comunica al sistema para generar una recomendación (incluye preferencias, gustos y necesidades del usuario).

c) Algoritmos para realizar la recomendación: combinan los datos de campo con los de entrada para generar recomendaciones solicitadas.

A la hora de obtener los datos de entrada podemos distinguir entre:

a) Información explicita: el usuario informa sobre sus preferencias (mediante una puntuación normalmente)

b) Información implícita: a partir de las acciones del usuario (historial de navegación, cookies,...) el sistema recogerá información mediante una aproximación de minería de datos.

\subsubsection{Sistemas de recomendación colaborativos}

Este tipo de técnica añade valoraciones a los objetos, identificando los gustos comunes de los usuarios basándose en sus valoraciones. Los resultados que proporcionan se basan en las valoraciones realizadas por usuarios con gustos similares al que realiza la petición de la valoración. La principal ventaja es que son independientes de la representación de los productos que se recomienden (Schwab et al, 2001). A su vez estos modelos de recomendación pueden ser clasificados en dos grupos:

a) Basados en modelos: antes de realizar una recomendación deben desarrollar un modelo basado en las calificaciones de los usuarios. El proceso de producción de modelos que usan los algoritmos se desarrolla con distintos enfoques de aprendizaje automático: redes bayesianas, encasillamiento, enfoques basados en reglas, etc.

b) Basados en memoria: utilizan la base de datos completa de usuarios-ítems para generar las predicciones. Mediante una técnica estadística se halla un conjunto de usuarios que tengan una historia de concordancia con el usuario objetivo (se denominarán vecinos). Se utiliza un algoritmo que combine las preferencias de los vecinos para producir una predicción y un ranking de los ítems a recomendar al usuario objetivo. 


\subsubsection{Sistemas de recomendación basados en contenido}

Se basan en categorizar lo ítems a recomendar, proporcionando resultados que tengan características similares con otros que han sido valorados anteriormente por el usuario (Bezerra, 2004).

Cada ítem llevará asociado un conjunto de atributos que lo describe. Analizando las características que ha valorado positivamente un usuario, se construye un perfil que es utilizado para buscar ítems que puedan satisfacer sus preferencias.

Como ventaja cabe indicar la sencillez de cara a la recolección de la información y a la aplicación de los algoritmos,

Como principal inconveniente de este método, cabe destacar, que acumulan bastante información sobre las preferencias de los usuarios que usan el sistema.

Otro problema de esta técnica es el estar limitado por las características asociadas que describen a los items que se pueden recomendar.

\subsubsection{Sistemas de recomendación demográficos}

En este sistema se clasifica a los usuarios según diferentes parámetros personales y se realizan las recomendaciones teniendo en cuenta el grupo demográfico al que pertenece el individuo. Se basa en el uso de estereotipos de usuarios (Pazzani, 1999).

Una de las principales ventajas de este sistema es que no requiere información histórica, requisito necesario en los sistemas colaborativos y basados en contenido.

El principal inconveniente de este sistema es que requiere información demográfica sobre el usuario, muchas veces de carácter personal (edad, nivel, cultural, sexo,...), lo que hace que el usuario pueda ser reticente a sus uso por la vulneración de su privacidad.

Otro inconveniente viene dado por la dificultad de disponer de los datos demográficos necesarios.

\subsubsection{Sistemas de recomendación basados en conocimiento}

Este tipo de sistemas dispone de información sobre como un ítem satisface a un usuario y establece una relación entre necesidad y recomendación. Las recomendaciones se basan en la inferencia sobre las necesidades y las preferencias del usuario (Burke, 2000).

Estos sistemas de recomendación deben tener información sobre los diferentes usuarios y los ítems que pueden recomendarse, para poder realizar recomendaciones a partir de los ítems que pueden satisfacer sus preferencias. Es muy habitual recomendar items similares a los que le gustaron al usuario con anterioridad.

Aunque este sistema de recomendación necesita grandes cantidades de información (valoraciones de visitas anteriores), no están basadas en valoraciones de otros usuarios.

Uno de los inconvenientes de este sistema es que las recomendaciones siempre serán similares, nunca se recomendará algo totalmente nuevo o suficientemente diferente a un usuario.

A pesar de los posibles inconvenientes de estos sistemas de recomendación, han tenido bastante éxito cuando se solicitan ítems de un único dominio (restaurantes, canciones,...).

Como estos sistemas ofrecen buenos resultados en demandas puntuales, son usados también como complementos en sistemas híbridos.

\subsection{Sistemas de recomendación aplicados al contexto}

La mayoría de los sistemas de recomendación actuales se centran en recomendar los ítems más relevantes para los usuarios, sin tener en cuenta la información contextual (Adomavicius, G. et al. 2010). Algunos investigadores están comenzando a sostener que la 
información contextual relevante es muy importante en los sistemas de recomendación, siendo necesario tenerla en cuenta en el proceso de la recomendación.

Existen algoritmos que incorporan la información contextual en el proceso de la recomendación:

- Pre-filtrado contextual.

- Post-filtrado contextual.

- Modelado contextual.

La mayoría de los sistemas no tienen en cuenta elementos contextuales como el lugar, la hora, la compañía, presencia de niños, etc. Tradicionalmente no se han usado a la hora de la realización de la recomendación.

\subsubsection{Definición de contexto}

De un modo genérico se define como: "condiciones o circunstancias que afectan a alguna cosa”, no obstante se han presentado más de 150 definiciones de este término en diferentes campos.

Veamos algunas de las definiciones de contexto en aquellos campos que están más directamente relacionados con los sistemas de recomendación:

a) En minería de datos: "eventos que caracterizan las etapas de la vida de un cliente y que pueden determinar cambios en sus preferencias, estado y valoración"

b) Personalización de comercio electrónico: "circunstancias que provocan una intención de compra realizada por parte de un cliente en una aplicación de comercio electrónico" Diferentes intentos de compra pueden dar lugar a diferentes tipos de comportamientos.

c) Sistemas móviles contextuales: inicialmente se definió como la ubicación del usuario, la identidad de las personas cercanas a él, los objetos a su alrededor y los cambios en estos elementos. Posteriormente se han añadido otros factores a esta definición: fecha, estación del año, temperatura,... Esta información contextual es fundamental para dar proporcionar una amplia gama de servicios basados en la localización.

\subsubsection{El contexto en los sistemas de recomendación}

Los sistemas de recomendación contextuales (CARS) se ocupan de modelar y predecir los gustos y preferencias del usuario, mediante la incorporación de información contextual al sistema de recomendación como categorías adicionales de datos (Adomavicius et al., 2010).

La predicción de interés de un ítem a un usuario se obtendrá mediante una función basada en tres variables: usuario, ítem y contexto.

Por el contrario los sistemas tradicionales que no tienen en cuenta el contexto decimos que trabajan en 2D pues sólo tienen en cuenta las variables, usuario e ítem.

\subsubsection{Paradigmas para incorporar contexto en los sistemas de recomendación}

El uso de la información contextual en los sistemas de recomendación se remonta a trabajos en los que se partía de la hipótesis de que incluir conocimientos sobre tareas del usuario en el algoritmo de recomendación podía mejorar los resultados.

Las diferentes técnicas para utilizar información contextual en procesos de recomendación pueden ser categorizadas en dos grupos:

a) Recomendación a través de consultas y búsquedas contextuales. Este ha sido utilizado por una gran cantidad de sistemas de recomendación turísticos y de móviles. 
b) Recomendación a través de preferencias y estimaciones contextuales. Este representa una tendencia más actual en la literatura de sistemas de recomendación basados en el contexto. Intenta modelar y aprender las preferencias del usuario observando la interacción de este $\mathrm{y}$ otros con el sistema u obteniendo las preferencias mediante retroalimentación a partir del uso de varias recomendaciones previas.

Según el momento en el que hacemos uso de los elementos contextuales para el filtrado de POI's podemos distinguir los paradigmas que se muestran en la figura 1:

(a) Pre-filtrado context.

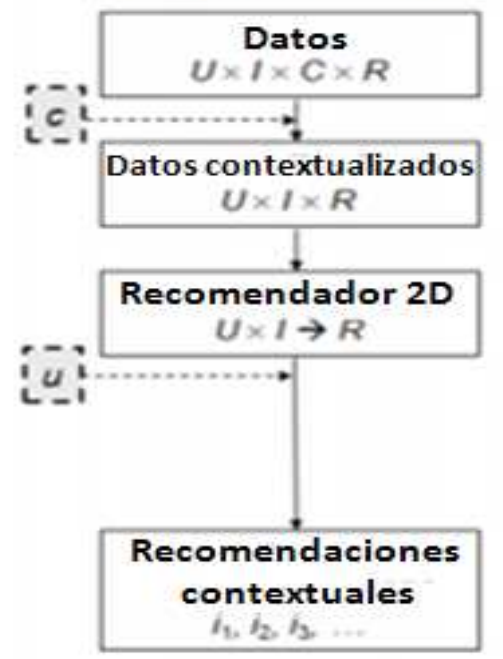

(b) Post-filtrado context.

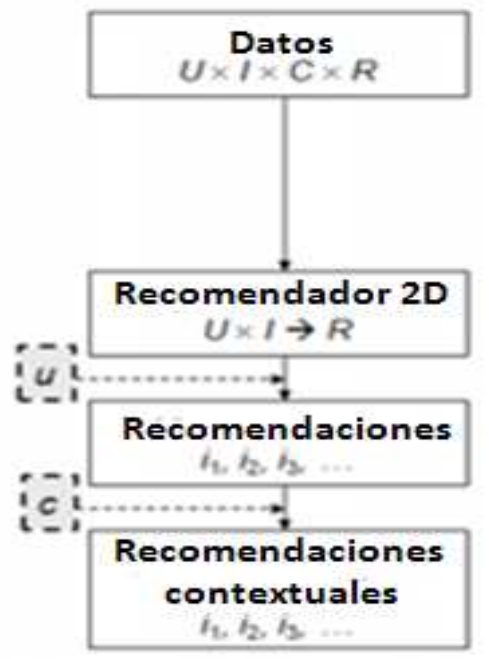

(c) Modelado context.

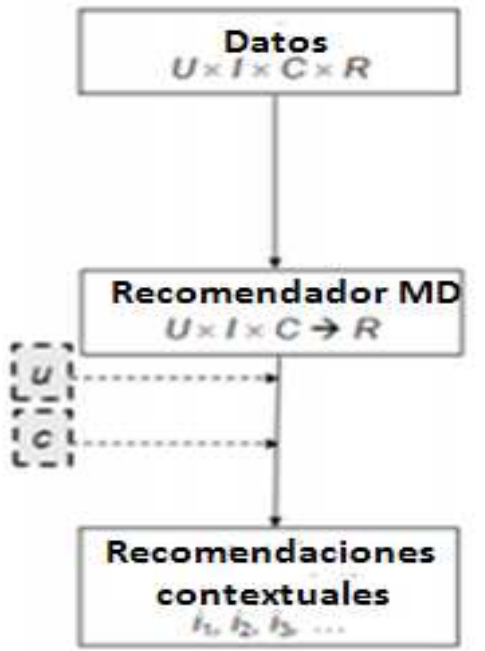

Figura 1. Diferentes opciones de aplicación del contexto

Claramente podemos ver que el pre-filtrado se aplica inmediatamente antes del tradicional algoritmo recomendador $2 \mathrm{D}$, haciendo que el conjunto de datos quede reducido a un conjunto igual o inferior de datos contextualizados.

El post-filtrado se aplica inmediatamente después del tradicional algoritmo recomendador $2 \mathrm{D}$, haciendo que las recomendaciones hechas por algoritmo recomendador sea filtrado obteniendo un conjunto igual o inferior de recomendaciones contextuales.

En cambio en el modelado contextual se está aplicando un algoritmo 3D donde se tienen en cuenta datos de usuarios ítems y contextuales.

\subsection{Técnicas de hibridación}

No existe un sistema de recomendación perfecto, todos poseen limitaciones, por lo que la técnica de hibridación es una solución para mejorar la eficiencia de las recomendaciones. Normalmente se mezclan dos o más técnicas de recomendación, para mejorar la precisión.

Las técnicas de hibridación más conocidas son:

a) Uso de pesos: combina las recomendaciones generadas por cada sistema de recomendación, asignando a cada uno de ellos un peso dependiendo del sistema que las genera.

b) Uso de técnicas "switch": este modelo alterna diferentes motores o técnicas dependiendo de la situación y el momento en el que se realiza la solicitud de la 
recomendación. La principal complejidad viene dada por la determinación del criterio por el que se utiliza un motor de recomendación u otro.

c) Mezclas: combina los resultados obtenidos por los diferentes sistemas de recomendación implicados. El principal problema es disponer de datos completos sobre las preferencias del usuario.

d) Cascada: el primer sistema obtiene unas recomendaciones que luego son refinadas por los siguientes sistemas. Tiene una gran eficiencia computacional.

\subsection{Recomendaciones para grupos}

Tradicionalmente los sistemas de recomendación vienen ocupándose de recomendar ítems a usuarios individuales.

En estos últimos años se ha empezado a trabajar en técnicas que permitan proponer recomendaciones a grupos de usuarios (Jameson, 2007). La mayoría de sistemas de recomendación grupales tienen métodos de adquisición de información distintos a los que se aplican a los sistemas individuales:

a) Adquisición de preferencias sin especificación explícita: el sistema funciona con información adquirida implícitamente sobre los usuarios.

b) Adquisición de preferencias explícitas: aquellos recomendadores en los que se necesita una especificación explícita de las preferencias de los usuarios.

Cuando el sistema tiene conocimiento sobre los diferentes usuarios, puede adoptarse la especificación de preferencias de la recomendación para el grupo.

Dependiendo de las características del grupo, como pueden ser tamaño, homogeneidad,...; puede resultar muy complejo encontrar una recomendación adecuada que satisfaga a todos los miembros. Lo más habitual es que el sistema escoja aquella opción que satisfaga al mayor número de usuarios de acuerdo a las preferencias individuales.

Es necesario encontrar métodos que permitan combinar información sobre sobre las distintas preferencias individuales de los usuarios, de forma que se pueda llegar a la recomendación idónea.

Podemos encontrar diferentes métodos:

a) Método simple: se mezclan recomendaciones individuales en una única lista.

b) Método de agregación: une las valoraciones para cada usuario y para cada ítem candidato. Se plantea como objetivo maximizar la satisfacción media, penalizando a aquellos que aunque tengan una media alta tienen alguna calificación baja por parte de algún miembro del grupo.

c) Método de construcción de perfil de grupo: se trata de la creación de un perfil especial para el grupo a partir de las características de todos los miembros del grupo.

La estrategia de agregación, frecuentemente utilizada, ha sido criticada ya que no tiene en cuenta las relaciones de los diferentes miembros del grupo con el resto. Para tener en cuenta la interacciones dentro del grupo podemos usar:

a) Recomendaciones basadas en la personalidad: por ejemplo, no todos los miembros de un grupo tienen la misma flexibilidad a la hora de aceptar una recomendación.

b) Recomendaciones basadas en factores sociales: se tienen en cuenta el grado de confianza que tiene un usuario con el resto. 


\section{PROTOTIPO DE RECOMENDACIÓN GRUPAL}

\subsection{Introducción}

A la hora de formalizar el prototipo de recomendación grupal, tenemos que abordar:

a) El tipo de sistema de recomendación a utilizar.

b) Si vamos a tener en cuenta el contexto o nos vamos a basar en un sistema 2D.

c) Qué algoritmo o algoritmos vamos a emplear para la recomendación de ítems a los usuarios.

d) Como resolver la cuestión de la recomendación grupal, según las distintas posibilidades que se han estudiado con anterioridad.

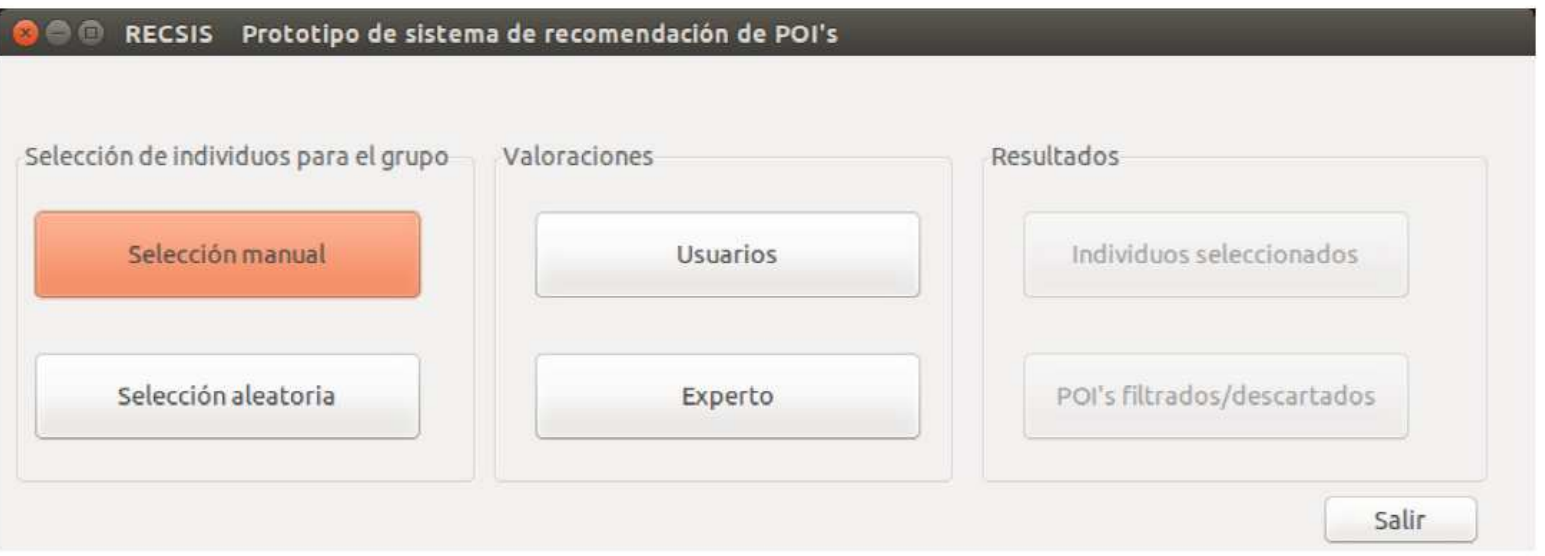

Figura 2. Pantalla principal del prototipo creado

\subsection{Sistema de recomendación utilizado}

Este prototipo se ha desarrollado básicamente como un sistema de recomendación basado en contenido. Se ha creado una base de datos de POI's ficticios cuyas valoraciones han sido realizadas por motores aleatorios que harían la función del experto del destino. El motivo es realizar pruebas con un número de puntos variable y grande.

En cuanto a las preferencias de los usuarios, se ha partido de una base de datos (generada también de forma aleatoria) en la que las preferencias de los usuarios aparecen ya registradas. Es decir, se supone que de alguna manera, valoraciones anteriores de los usuarios en otras visitas han sido recopiladas y almacenadas en la presente base de datos. Dadas las restricciones establecidas por la LOPD, aunque en un principio se pensó en recopilar esas preferencias a partir de un extenso grupo de alumnos universitarios, se optó por la generación de un conjunto de nombres y apellidos aleatorios y se hicieron las valoraciones de los usuarios a partir de una generación de datos aleatoria.

No obstante, estas valoraciones de los usuarios así como las valoraciones de los expertos, pueden ser modificadas de manera manual por parte del usuario del prototipo de cara a poder realizar distintas pruebas y comprobar que los resultados obtenidos son en mayor o menor medida razonables.

Como partimos de valoraciones realizadas por usuarios, entendemos que la información será lo suficientemente imprecisa para no usar una descripción cuantitativa. Por ello hemos optado por el uso de valores difusos en cuanto a las posibles valoraciones de los turistas. Del mismo modo, y como consecuencia de querer aplicar idéntica escala a las 
valoraciones de los expertos, hemos aplicado las mismas escalas difusas a las valoraciones de las características, atributos y descriptores de los POI's.

Para resumir de un modo sencillo a que nos referimos cuando hablamos de escalas difusas nos valdremos de un ejemplo: supongamos una escala para definir la juventud o madurez de un individuo formada por los siguientes valores:

- Joven.

- Maduro

- Anciano

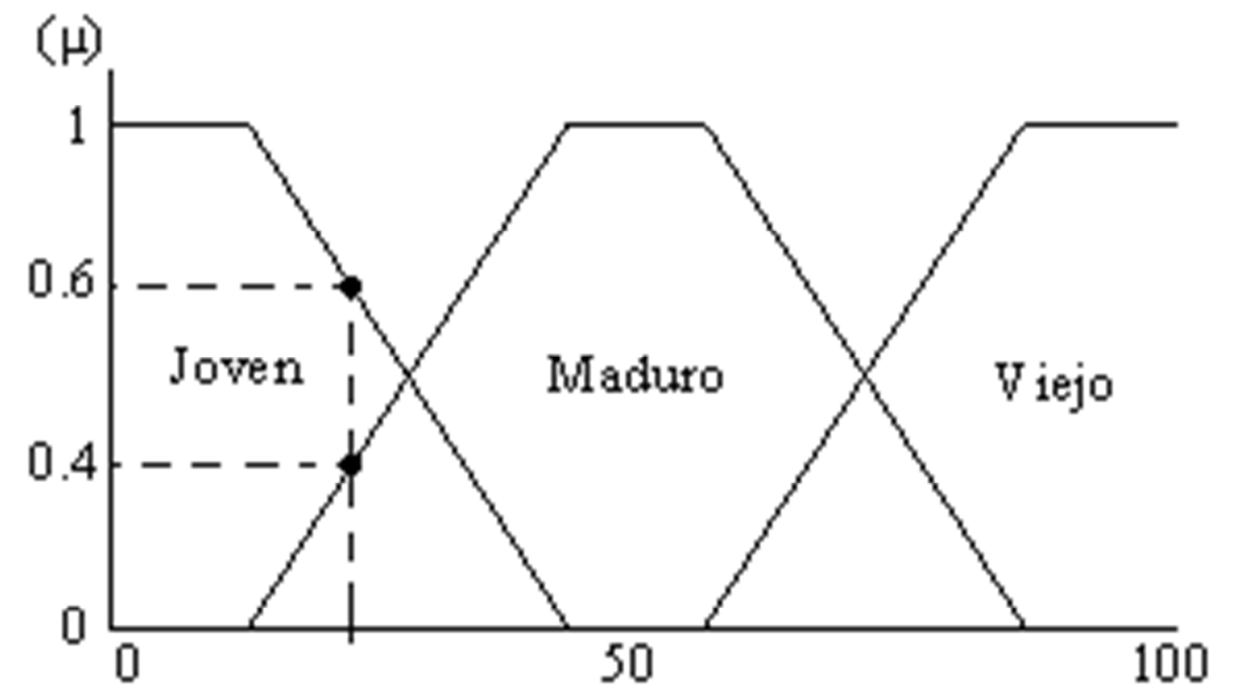

Figura 3. Valores difusos

Según los criterios actuales todo el mundo estará de acuerdo en que una persona de 5 años es joven una de 50 es madura y una de más de 90 es anciana. No obstante si una persona de 30 años podría ser considerada para algunos como madura, para otros como joven y habrá quien piense que haría falta una nueva categoría.

Pues bien esto es un ejemplo de una escala difusa. Y en este tipo de escalas nos basaremos de cara a la valoración tanto de los expertos como de los usuarios. 


\section{(C) Mantenimiento de tabla: escalas}

\begin{tabular}{|c|c|c|c|c|c|c|c|c|}
\hline Id: 1 & Nom & bre: & Escala 1 & & & No valores: & & \\
\hline Etiqueta1 & $\mathrm{Si}$ & & Valoría & 0.5 & Valorib & 1 & Valoric & 1 \\
\hline Etiqueta2 & Indiferente & & Valorza & 0 & Valor2b & 0.5 & Valor2C & 1 \\
\hline Etiqueta3 & No & & Valor3a & 0 & Valor $3 b$ & 0 & Valor $3 \mathrm{C}$ & 0.5 \\
\hline Etiqueta4 & & & Valor4a & & Valor $4 b$ & & Valor $4 c$ & \\
\hline Etiqueta5 & & & valor $5 a$ & & Valor $5 b$ & & Valor $5 \mathrm{C}$ & \\
\hline Etiqueta6 & & & Valor6a & & Valor $6 \mathrm{~b}$ & & Valoróc & \\
\hline Etiqueta7 & & & Valor7a & & Valor7b & & Valor7c & \\
\hline Etiqueta8 & & & Valor8a & & Valor8b & & Valor Bc & \\
\hline Etiquetag & & & Valor9a & & Valorgb & & valorge & \\
\hline Añadir & Eliminar & & Modifica & & vardar & Cancelar & & Salir \\
\hline Id & Nombre & Nume & erovalor & Etiqueta1 & Etiqueta2 & Etiqueta3 & Etiqueta 4 & Etiquet: \\
\hline 1 & Escala 1. & 3. & & si & Indiferente & No & & \\
\hline 2 & Escala 2 & 5 & & Excelente & Mucho & Normal & Poco & Nada \\
\hline 3 & Escala 3 & 7 & & Totalmente & Casi todo & Más de lo nor & ri Normal & Menose \\
\hline 4 & Escala 4 & 5 & & $\mathrm{Si}$ & Bastante & Normal & Poco & Nada \\
\hline
\end{tabular}

Figura 4. Acceso a la pantalla de valoraciones del prototipo

\subsection{Aplicación de atributos contextuales}

Vista toda la documentación mencionada al respecto de los sistemas de recomendación, no parecía razonable no tener en cuenta la aplicación de los atributos contextuales a la hora de la realización del presente prototipo.

Teniendo en cuenta las tres opciones disponibles en los sistemas en los paradigmas contextuales aplicados en sistemas de recomendación, en este prototipo se ha optado por el pre-filtrado contextual. 
(a) Pre-filtrado context.

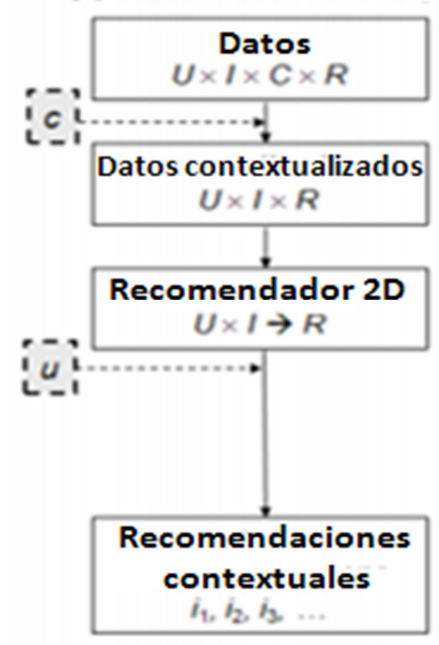

Figura 5. Selección del mecanismo de filtrado contextual

Los motivos que han llevado a esta decisión han sido:

- Con respecto al filtrado post-contextual, el sistema recomendador partirá de un conjunto de datos menor (o a lo sumo igual) que el de los datos no filtrados. Lo cual a la hora del coste computacional y de la cantidad de registros a tratar en grandes conjuntos de datos hará que el sistema sea más rápido.

- Con respecto al recomendador con modelado contextual, esta opción se presenta mucho más sencilla, de cara a los algoritmos a utilizar y de cara al coste computacional que pueden tener estos algoritmos más complejos y el conjunto de datos también más amplio..

\subsection{Algoritmo utilizado}

El algoritmo utilizado se resume a continuación:

Paso 1: Se realiza el filtrado contextual ${ }^{5}$, no sólo en lo referente a la distancia del grupo a los POI's, sino también en lo relativo a la presencia de niños o discapacitados en el grupo, el horario y día de la semana planeado, el deseo por parte de los miembros del grupo de comer en el POI o sus proximidades, y la previsión meteorológica del día seleccionado para la visita.

Evidentemente toda esta información de carácter contextual ayudará a seleccionar o descartar cada uno de los POI's existentes en la BBDD y nos generará dos nuevas tablas poisFiltrados y poisDescartados. A partir de la primera de ellas continuaremos con el paso 2 del presente algoritmo.

Para el cálculo de las distancias dadas las posiciones del grupo y del POI mediante coordenadas de longitud y latitud se ha usado el algoritmo de "haversine". Para el resto de los atributos contextuales se analiza si el POI en cuestión está abierto o no en el día y hora de la visita, si la presencia de niños o discapacitados es un impedimento para la visita, si existen lugares en el entorno para poder comer y las condiciones meteorológicas que puedan ser adversas para la visita.

5 Los datos correspondientes al filtrado contextual no son tomados de la tabla de valoraciones de los usuarios independientes. Por comodidad se ha optado por preguntar por los atributos contextuales en el momento de seleccionar los POI's. 
Normalmente todos estos ítems van a tener tres posibles valores:

- Sí

- Indiferente

- No

Veamos con un par de ejemplos como se hace el filtrado:

- Sea un grupo en el que hay niños. Entonces tendremos la casilla correspondiente a niños en el grupo, marcada. El algoritmo rechazará todos aquellos POI's en los que se indica que la presencia de niños "No" es recomendable y pasará al grupo de los filtrados con éxito todos aquellos en los que la presencia de niños haya sido valorada por los expertos como "Sî" o "Indiferente".

- De manera análoga si un grupo de turistas solicita una recomendación para visitar POI's un día que "Sî" hay previsión de lluvia, descartará todos aquellos POI's que expresamente hayan sido declarados por el experto como "No" recomendables con lluvia y admitirá todos aquellos POI's que hayan sido calificados como "Sí" o "Indiferente". No obstante si la previsión de lluvia no es clara y es marcada como indiferente, todos los POI's pasarán el filtrado con éxito dependiendo de la calificación dada por el experto.

\section{Recomendación de POIS. Paso 1,2 y 3 busqueda de incompatibilidades.}

Atr. contextuales del grupo:

Niños en el grupo

$\square$ Discapacitados en el grupo

$\square$ Comer en el POI

Atr. contextuales de lugar:

Longitud:

Latitud:

Radio visita:

\section{$-4.5038835$}

36.6240771

200

$\mathrm{km}$

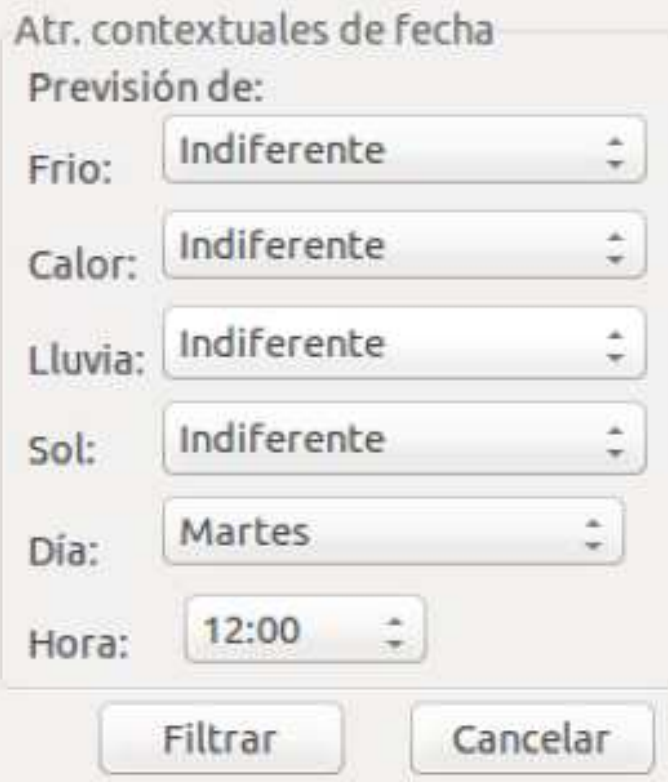

Previsión de:

Frio: Indiferente
Calor: Indiferente
Lluvia: Indiferente
Sol: Indiferente
Día: Martes
Hora: $12: 00:$
Filtrar $\quad$ Cancelar

Figura 6. Pantalla de filtrado contextual del prototipo

Tanto en éste paso como en los siguientes, se han definido unas operaciones muy sencillas para comparar las valoraciones de las características de los puntos tanto con las características contextuales como con las individuales de cada miembro del grupo:

- Si la etiqueta del POI coincide con la valoración contextual del grupo o la particular del usuario le daremos un valor 1 . 
- Si la valoración del POI y del usuario son contiguas en la escala le daremos una valoración 0,5.

- En otro caso daremos una valoración 0 .

Evidentemente, las escalas aplicadas a los POI's se han hecho coincidir con las correspondientes aplicadas al contexto y las valoraciones de los usuarios. Podriamos haber utilizado diferentes escalas para lo cual necesitaríamos operaciones más complejas para poder hacer las comparaciones oportunas (Galindo et al, 2008).

Paso 2: A partir de los POI's filtrados que ya han sido almacenados en la tabla poisFiltrados tras el paso 1, calculamos el valor de similitud para todos los usuarios del grupo con respecto a los descriptores y características seleccionados para cada punto de interés que pasó el filtro con éxito:

$$
\operatorname{Sim}(N(u), D s(i))=\operatorname{Sim}_{i}^{u}
$$

A partir de los valores calculados con esta fórmula construimos una matriz, donde tendremos, las valoraciones de todos los POI's filtrados respecto a los atributos (descriptores y características) que fueron valorados en su momento por los expertos (descriptores y características de los POI's) y por los usuarios (descriptores y características de los usuarios).

$$
\forall u, \forall i \operatorname{Sim}=\left(\begin{array}{ccc}
\operatorname{Sim}_{11} & \ldots \operatorname{Sim}_{1 j} \ldots & \operatorname{Sim}_{1 n} \\
\square & \square & \square \\
\operatorname{Sim}_{n 1} & \ldots \operatorname{Sim}_{n j} \ldots & \operatorname{Sim}_{n m}
\end{array}\right)_{7}
$$

Paso 3: Construimos la matriz de preferencia de cada usuario. A partir de las medidas de similitud calculadas en el paso anterior, se obtiene la relación de preferencia para cada POI. Para ello partimos del grado de inclusión (Zadeh, 1996). Es una medida que considera un POI A como referencia y mira las características de otro POI B que son comunes. Este grado se denota como S(A,B) y su cálculo se obtiene de la siguiente forma:

$$
S(A, B)=\inf _{x} \min \left(1-F_{A}(x)+F_{B}(x), 1\right)
$$

Donde $\mathrm{F}_{\mathrm{A}}(\mathrm{X})$ y $\mathrm{F}_{\mathrm{B}}(\mathrm{X})$ son las funciones de pertenencia de $\mathrm{A}$ y $\mathrm{B}$. De modo que para obtener el grado de preferencia del POI A sobre el POI B para un usuario dado tendremos:

$$
\operatorname{pref}_{A B}=S(B, A)
$$

Por tanto nuestro algoritmo recorrerá todos los POI's comparando todos los atributos (descriptores y características). Como necesitamos saber el grado de preferencia entre un punto " $\mathrm{i}$ " y un punto "j" por parte de un usuario " $\mathrm{u}$ " necesitamos tener calculado los valores de similitud $\operatorname{Sim}_{i}^{u}$ y $\operatorname{Sim}_{j}^{u}$ para cada un de los usuarios (estos valores son los que obtuvimos precisamente en el paso 2). Por tanto, ahora para cada usuario, calculamos la matriz de preferencia entre cada dos puntos $\mathrm{i}, \mathrm{j}$ de los POI's previamente filtrados:

6 Valor de similitud para el usuario "u" y el punto de interés "i". N(u) hace referencia a la necesidad del turista "u" y Ds(i)hace referencia al conjunto de características, atributos y descriptores del punto de interés "i".

7 Se construye una matriz en la que se tienen los valores de similitud para todos los pares de usuario - punto de interés. 


$$
\operatorname{pref}_{i j}=S(j, i)=\inf _{x} \min \left(1-F_{\operatorname{Sim}_{j}^{u}}(x)+F_{\operatorname{Sim}_{i}^{u}}(x), 1\right)_{8}
$$

a partir de la aplicación de esta fórmula para cada dos puntos obtendremos la matriz de preferencia en cuestión:

$$
\operatorname{PREF}_{u}=\left(\begin{array}{ccc}
\operatorname{pref}_{11} & \ldots \text { pref }_{1 j} \ldots & \text { pref }_{1 n} \\
\square & \square & \square \\
\operatorname{pref}_{n 1} & \ldots \text { pref }_{n j} \ldots & \text { pref }_{n n}
\end{array}\right)
$$

Una vez aplicado el algoritmo tendremos una matriz de preferencia para cada uno de los usuarios del grupo.

Paso 4: Calcular la matriz de preferencia estricta de cada usuario para cada punto o actividad turística, donde cada elemento se calcula de la siguiente manera:

$$
\text { pref }_{i j}^{e s t r i c t a}=\operatorname{máx}\left(\text { pref }_{i j}-\text { pref }_{j i}, 0\right)_{9}
$$

A partir de estos valores construimos la matriz de preferencia estricta:

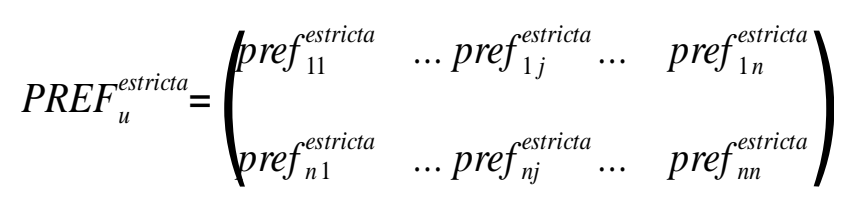

Paso 5: Cálculo del grado de no dominancia (GND), que indica el grado en el que un ítem no es dominado por una mayoría difusa del resto (Pérez, 2008), con lo que debemos calcular para cada POI, este valor, para lo cual usamos la siguiente fórmula:

$$
G N D_{i}=\min \left\{1-\text { pref }_{j i}^{\text {estricta }}, j \neq i\right\}_{10}
$$

Paso 6: Para cada POI se obtiene la suma de los valores obtenidos de forma individual por cada uno de los miembros del grupo $G N D_{i}^{g}$ y de este modo obtendremos un conjunto ordenado de POI's recomendados.

8 Calcularemos el valor mínimo, que será siempre inferior o igual a 1, relativo a la preferencia que tiene un usuario "u" a un punto de interés "i" con respecto a un punto de interés "j". Esto lo haremos teniendo en cuenta todos los descriptores y características de los puntos en cuestión.

9 Se calcula la matriz de preferencia estricta de cada usuario a partir de la matriz de preferencia calculada con anterioridad., el resultado estará siempre entre 0 y 1.

10 Mediante el uso de la matriz de preferencias estrictas de cada usuario calculamos los grados de no dominancia de cada punto de interés, 


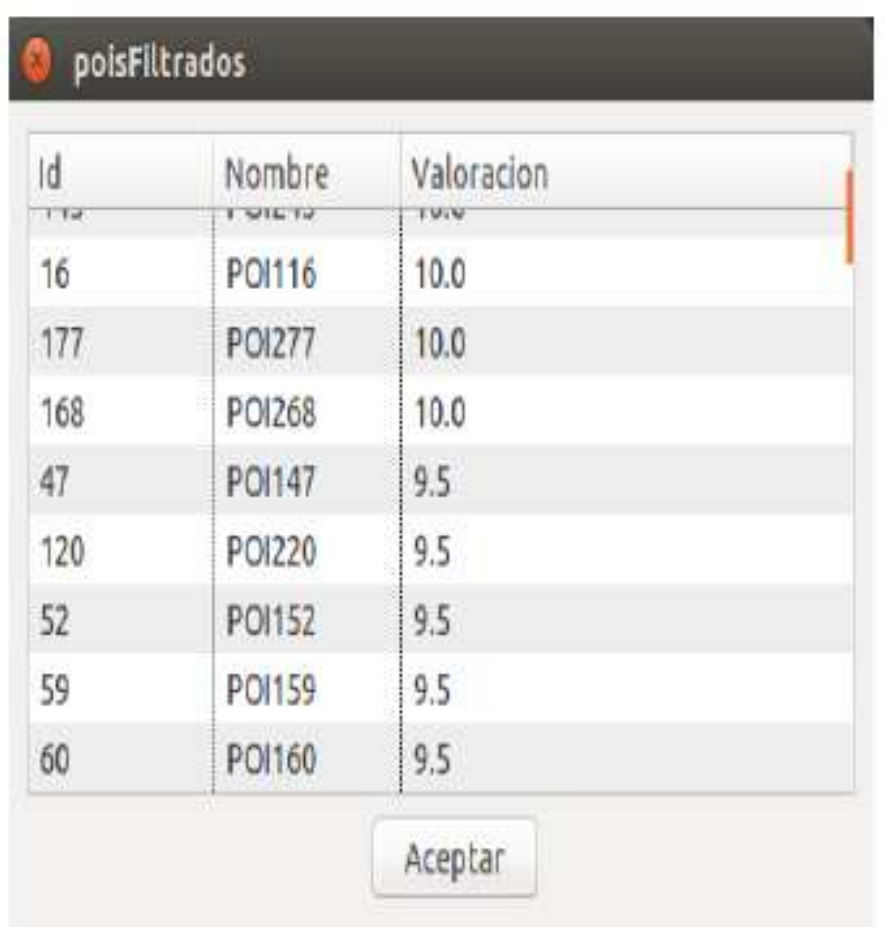

Figura 7. Pantalla de muestra de recomendaciones del prototipo

\subsection{Sistema de recomendación grupal}

Aunque el objetivo de un sistema real sería mostrar un conjunto reducido de POI's que serían los candidatos mejor recomendados para el grupo en cuestión, al tratarse éste de un prototipo no se ha querido truncar la lista de todos los POI's previamente filtrados en el paso 1, lo que permite mostrarlos todos con su grado de no dominancia para el grupo en cuestión.

Aunque habría otros factores a tener en cuenta para la recomendación grupal cuando la satisfacción de todos los miembros del grupo no es plena, estos no han sido tenidos en cuenta. Se podría haber maximizado la satisfacción media del grupo teniendo en cuenta un factor de dominancia de la personalidad de los miembros del grupo.

Podríamos también haber minimizado la carencia, de modo que aunque la satisfacción media sea alta, si un POI i tiene un GND muy bajo para un usuario $u$ perteneciente al grupo, podríamos descartarlo.

Dado que existen diversos puntos de vista al respecto y no hay un consenso sobre cual o cuales serían las lineas a seguir en estos casos, se ha decidido terminar el prototipo mostrando los resultados tal cual se obtienen tras el paso 6 del algoritmo.

\subsection{Resultados}

Aunque el objetivo de un sistema real sería reducir el número de POI's de cara a facilitar la selección por parte del grupo (o del líder que dirige a dicho grupo), a efectos de estudio en el presente prototipo, se maneja una lista de resultados mayor ${ }^{11}$ para poder comparar distintas soluciones con la aplicación de diferentes fórmulas y/o grupos.

11 De hecho se mostrará la lista de todos los POI's que hayan pasado el filtrado contextual mostrando la valoración obtenida. 
Por supuesto, los resultados arrojados por el presente prototipo, pueden servir además de para el estudio de las fórmulas empleadas y posibles resultados aplicados a grupos más o menos heterogéneos, para ser un punto de partida para aplicaciones de realidad aumentada o para algoritmos de búsqueda de POI's en distintas áreas geográficas.

El prototipo presentado en este proyecto está pensado para recomendar POI's a grupos, no obstante puede ser empleado para recomendar POI's a un turista que viaja sólo ${ }^{12}$.

Para que la aplicación funcione de manera correcta es necesario que el o los expertos hayan valorado cada uno de los descriptores, características y atributos contextuales de cada POI. También es necesario que tengamos los descriptores y características de todos los usuarios del grupo.

\section{CONCLUSIONES}

La implantación de herramientas innovadoras aplicadas al turismo como pueden ser las basadas en sistemas de recomendación potencia al sector turístico, pero no aportará todas sus ventajas potenciales si no se integran con los sistemas ya existentes. La interoperabilidad de los diferentes sistemas de un destino turístico permite que la gestión de la información sea sostenible y con un nivel de calidad satisfactorio, lo que redundará en beneficio de las empresas, destinos turísticos y usuarios.

El turista de nuestros días está habituado a las tecnologías de la información y demanda de ellas respuestas, también en el ámbito turístico. Las empresas y las instituciones relacionadas con el turismo deben proporcionar herramientas para prestar un mejor servicio y ofertar aquellos productos que son más afines al turista.

En este artículo se presenta un prototipo de recomendación para grupos que tiene en cuenta el contexto y que está basado fundamentalmente en contenido. El prototipo presentado en este trabajo está pensado para turistas que viajan en grupo, aunque podría utilizarse para un turista que viaja sólo.

El uso de información difusa resulta muy interesante ya que permite al turista especificar sus preferencias con grado de libertad grande y con un lenguaje cercano. Es muy importante, tener en cuenta, que para que el presente sistema pueda funcionar se necesita una recopilación previa de las preferencias de los turistas, así como las valoraciones de los expertos.

El turista actual demanda cada vez más información adaptada a sus preferencias, por lo que las empresas y las instituciones de gestión del turismo y de destinos deben proporcionar herramientas para prestar un mejor servicio y ofertar aquellos productos que son más afines al turista. Sistemas como el propuesto en este trabajo facilitarán a las propias empresas y organizaciones de destinos obtener el conocimiento y preferencias de los turistas, la demanda y trazabilidad, pudiendo conocer de manera eficiente la realidad del destino así como orientar la política turista.

En el trabajo futuro creemos puede resultar interesante que cada usuario que forme parte del grupo pueda conocer el motivo por el que el sistema ofrece esa recomendación, y cómo es de atractiva para cada miembro del grupo.

12 Bastaría con que seleccionáramos un grupo de un sólo turista y obtendríamos el resultado para ese grupo unitario. 


\section{REFERENCIAS BIBLIOGRÁFICAS}

Adomavicius,G.; Tuzhilin,A.; Berkovsky,S. \& Said A. (2010). "Context-awareness in recommender systems: research workshop and movie recommendation challenge". RecSys 2010: 385-396

Aguayo, A.; Guevara, A.; Rossi, C.; Caro, JL. \& Leiva, JL. (2010) "Sistema integrado de gestión de destinos". Congreso Turitec 2010. Málaga.

Batet, M., Moreno, A., Sánchez, D., Isem, D \& Valls, A. (2012) “Turist@: Agent-based personalised recommendation of tourist activities": Expert Systems with Applications, Volume 39, Issue 8, 2012: 7379-7329

Bernardos, A.B. (2007) "Servicios y aplicaciones en movilidad para el sector turístico". CITIC, Madrid

Bezerra, B. \& Carvalho, F. (2004). "A symbolic approach for content-based information filtering”. Information Processing Letters, 92 (1)

Borràs, J., Moreno, A., Valls, A., Ferré, M., Ciurana, E., Salvat, J. Russo, A.P. \& AntonClavé, S. (2012) "Uso de técnicas de inteligencia artificial para hacer recomendaciones enoturísticas personalizadas en la provincia de Tarragona". Congreso Turitec 2012. Málaga.

Burke, R. (2000). "Knowledge-based recommender systems". Encyclopedia of Library and Information Systems, 69 (32)

Castejón, R. \& Méndez, E. (2012): “Introducción a la economía para turismo”. Prentice-Hall. España

Fesenmaier, D.R.; Werthner, H. \& Wober, K. (2006) "Destination Recommendation System. Behavioural Foundations and Applications", CAB International, USA.

Galindo, J., Urrutia, A. \& Piattini, M. (2006). "Fuzzy databases, modeling, design and implementation”. Ed. Idea Group Inc (IGI)

Goh, D.; Lee, C \& Ang, R. (2010) "Determining Services for the Mobile Tourist". The Journal of Computer Information Systems, 51 (1): 31-40.

Jakkilinki, R.; Georgievski, M. \& Sharda, N. (2007). "Connecting Destinations with Ontology Bases e-Tourism Planner". Information and Communication Technologies in Tourism.Ed.Springer: 21-32.

Jameson, A. \& Smyth, B.(2007) "Recommendation to groups" The Adaptive Web, Methods and Strategies of Web Personalization (editado por P. Brusilovsky, A. Kobsa y W. Nejdl), vol. 4321 de Lecture Notes in Computer Science, Spriger: 596-627

Lamsfus, C.; Alzua-Sorzabal, A.; Martín, D.; Salvador, Z. \& Usandizaga, A. (2009) "HumanCentric Semantic-bases Context Modelling” In Tourism. Mediterranean Conference on Information Systems. Athens, Greece.

Lashkari, A., Parhizkar, B. \& Mohamedali, M.A. (2010). "Augmented Reality Tourist Catalogue Using Mobile Technology"

Leiva, J.L. (2014), "Realidad aumentada bajo tecnología móvil basada en el contexto aplicada a destinos turísticos". Tesis Doctoral. Universidad de Málaga.

Leiva, J.; Guevara, A. \& Rossi, C. (2012) "Sistemas de recomendación para realidad aumentada en un sistema integral gestión de destinos". Revista de Análisis Turístico, no 14: 69-81

Lymberopoulos, D., Zhao, P., König, A., Berberich, K. \& Liu, J. (2011). "Location-aware click prediction in mobile local search".

Pazzani M.J. (1999). "A framework for collaborative, content-based and demographic filtering”. Artificial Intelligence Review, 13: 393-408.

Pérez, L.G. (2008), "Modelo de recomendación con falta de información. Aplicaciones al sector turístico". Tesis doctoral. Universidad de Jaén.

Peters, J. (2009) "Ten technology advances that will change air travel" A new frontier paper, SITA.

Schwab I.; Kobsa A. \& Koychev I. (2001). "Learning user interests through positive examples using content analysis and collaborative filtering". Technical report, Fraunhofer Institute for Applied Information Technology, Germany, 2001. 
Schafer, J.; Frankowski, D.; Herlocker, J. \& Sen, S.(2007) "Collaborative Filtering Recommender Systems”. In Adaptive Web 2007, LNCS, vol. 4321, Heidelberg, Springer: 291-324

Sundbo, J.; Orfila, S. \& Sorensen, F. (2007)."The innovative behaviour of tourism firms. Comparative studies of Denmark and Spain”. Research Policy, no88: 88-106

UNWTO (2015). "UNWTO, Compendium of Tourism Statistics, Data 2009-2013"

Xiang, Z. \& Pan, B. (2011): "Travel queries on cities in the United States: Implications for search engine marketing for tourist destinations.” Tourism Management, 32: 88-97

Zadeh, L.A. (1996). "Fuzzy logic = computing with words". IEEE Transactions on Fuzzy Systems, 4(2):103-111

\section{HOW TO CITE THIS ARTICLE IN BIBLIOGRAPHIE}

Vargas Pérez, P. y Leiva Olivencia, J. L. (2015): "Prototipo de sistema de recomendación grupal en un destino turístico" Rotur. Revista de Ocio y Turismo, 9: 62-81, http://www.rotur.es, ISSN: 1888-6884 